pouvoirs, M. Wassiltchikoff, un nouveau médecin-chirurgien fut encore envoyé de St-Pétersbourg au Monténégro, et quelques semaines après (15/27 février), un troisième convoi de matériel sanitaire pesant 216 pouds (près de 4,000 kilog.), put être expédié pour faire face aux besoins. Comme les offrandes en toute espece de matériel sanitaire ont afflué dans les dépôts de la Société russe de la Croix rouge, provenant de toutes les classes et provinces de l'empire, un quatrième envoi pourra sous peu suivre les autres.

Les dépenses prélevées sur les fonds de notre Société de la Croix rouge el occasionnées par l'expédition de notre personnel de santé et son entretien au Monténégro, se couvrent aisément par les nombreux dons pécuniaires recueillis par les 176 comités locaux, éparpillés sur l'immense territoire de l'empire. Le total de ces dons est aujourd'hui de 45,000 roubles.

\title{
SUĖDE
}

\section{LA SOCIÉTÉ SUÉDOISE EN 1875}

Compte rendu présenté par le Comilé central à l'assemblée générale $d u 1^{\text {er }}$ novembre 1875

"Aujourd'hui que l'Association de secours aux militaires blessés et malades en temps de guerre se réunit en assemblée générale, le Comité central a le devoir, en vertu du paragraphe 11 des règle ments, de présenter à l'Association un exposé succinct de ses travaux pendant l'année qui vient de s'écouler.

Il est facile de comprendre qu'à une époque où la plupart des nations européennes paraissent se vouer à la paix et aux travaux pacifiques, I'Association de secours aux militaires blessés et malades en temps de guerre, laquelle fait toujours penser à l'éventualité d'une guerre, semble n'avoir qu'un but éloigné qui ne saurait ni 
mériter des sympathies ni exiger des sacrifices. De là vient le peu d'intérêt que le grand public voue en temps de paix à des associations pareilles. Mais le Comité central voit les choses à un autre point de vue; il croit que ces associations doivent et penvent, précisément en temps de paix, travailler à améliorer et à développer le service de santé militaire. Sub pace para bellum. Il s'agit d'étudier le matériel et les moyens de transport avec les améliorations qui y ont été apportées, puis de s'approprier les connaissances et l'expérience acquises dans les divers pays. Le Comité a cherché à travailler dans ce but.

Pour ce qui est de l'organisation intérieure du Comité central, nous devons noter les changements survenus par suite du décès du major-général D. W. Silfverstolpe qui, malgré son grand âge, avait, avec le plus grand zèle et le plus vif intérêt, pris part à tous les travaux du Comilé depuis le premier moment de la fondation de l'œuvre. A sa place, le Comité a éln vice-président, un de ses membres suppléants, M. le docleur S.-R.-D.-K. Olivecrona, conseiller à la Cour suprême ; et membre suppléant, en remplacement de M. Olivecrona, M. le docteur G.-J. Holmstròme, médecin en chef au régiment des dragons de la garde royale. Ces deux messieurs ont accepté ces charges respectives. De plus, M. A.-G. von Heyne, auditeur au régiment de la garde royale à cheval, a élé noumé secrétaire du Comité, en remplacement de M. le capitaine d'artillerie F. de Ron, qui est en service hors de la ville de Stockholm.

L'instruction des garde-malades a été continuée à l'hôpital d'Upsal, sous la direction si zélée et si intelligente de Mademoiselle F. Rappe. Le nombre des garde-malades ainsi formées s'élève aujourd'hui à 66, dont 44 élevées au dit hòpital. Ces garde-malades qui sont tenues, par des contrats passés avec l'Association, de se mettre à sa disposition, dans le cas où la Suède serait entraînée à faire la guerre, sont répandues dans les diverses parties du pays et $y$ font beaucoup de bien, soit par les soins qu'elles offrent aux malades, soit par les conseils et avis généraux qu'elles donnent, et par lesquels elles préviennent bien des maladies.

Pour qu'en cas de guerre l'action de l'Association soit déterminée d'avance, le Comité central a, dans sa séance du 28 octobre dernier, voté ce qui suit : 
L'Association de secours en temps de guerre, à laquelle il n'appartient pas de travailler isolément, mais conjointement avec le service de santé officiel, et sous la direction supérieure de l'administration générale de ce mème service, doit, lorsque la Suède et la Norwège se trouvent engagées dans une guerre, prier le gouvernement, par l'entremise du Comité central, de déléguer un commissaire royal, dont l'autorité serait déterminèe par des instructions spéciales. Ce commissaire servira d'intermédiaire entre le commandant en chef de l'armée et l'Association; celle-ci aura à se conformer aux instructions qui lui seront données.

\section{§ 2 .}

- Les places devenues vacantes au sein du Comité central, par suite de la guerre, seront repourvues aussitòt que faire se pourra; de plus, il sera au besoin procédé à l'élection de nouveaux membres.

\section{$\$ 3$.}

Lorsque les comités subdivisionnaires auront fait connaitre les ressources en personnel et en matériel dont ils disposent, le Comité central devra fournir au commissaire royal un compte rendu, aussi complet que possible, des ressources mises à sa disposition, en lui indiquant dans quelle mesure on peut compter sur ces ressources.

\section{$\$ 4$.}

La localité où le commissaire royal établit ses bureaux est considérée comme le siége de l'Association. L'action de l'Association a principalement pour but:

a) De procurer aux hôpitaux établis derrière les lignes des garde-malades bien instruites et des personnes propres au transport des blessés et malades des hôpitaux de campagne;

b) De réunir, expédier et distribuer, par l'entremise de ses délégués, les dons volontaires;

c) De venir en aide aux hôpitaux établis derrière les lignes, soit en acceptant certaines parties de l'administration, soit en soignant les convalescents, soit en établissant des hòpitaux spéciaux;

d) De servir d'intermédiaire entre les blessés et malades et leurs parents.

En vue de ces divers buts assignés à l'activité de l'Association, le Comité doit, en temps de guerre, distribuer entre ses divers membres le soin de ces diverses affaires. 


\section{1}

$\S 5$.

Le Comité, qui est considéré comme permanent pendant toute la durée de la guerre, procède à la quête d'argent et de matériel, qui seront expédiẻs aux destinations marquées. Le Comité doit en rendre compte.

Dans l'appel que le Comité adresse au public par la voie des journaux, ou par toute autre voie, il faut en premier lieu demander de l'argent, puis des bandages, de la charpie, du linge neuf et vieux, des matelas, des rafraichissements, etc.

Le Comitè priera S. Exc. M. le ministre des affaires étrangères de faire parvenir, par l'entremise des légations, l'appel du Comité aux nationaux établis à l'étranger. Le Comité en fera également part au Comité international à Genève, en y joignant les indications nécessaires d'adresse, de moyens d'envoi, etc.

\section{$\S 6$.}

Le Comité doit, par l'entremise du commissaire royal, chereher à obtenir, pendant la durée de la guerre, libre passage sur les chemins de fer et les bàtiments de transport de l'Etat, ainsi que l'envoi gratis de ses lettres et dépêches télégraphiques.

\section{$\$ 7$.}

Les insignes de l'Association doivent êtres munis du cachet du commissaire royal.

$\S 8$.

Tout employé salarié de l'Association est muni d'un livret, qui contient les conditions d'admission de l'employé et sert de livre de comptabilité pour les payements faits à cet employé. Dans ce livret sont inscrites, en outre, les instructions données au porteur.

$\$ 9$.

Le Comité doit, de temps à autre, rendre compte dans les journaux des dons et de leur emploi.

$\$ 10$.

En établissant des hôpitaux ou d'autres locaux aux frais de l'Association, le Comité doit toujours avoir en vue l'expérience acquise dans les autres pays.

$\S 11$.

L'ambulance de l'Association est mise en état de fonctionner. 
Un membre du Comité, M. le docleur Edholn, médecin militaire en chef, ayant déclaré à la réunion du 15 février dernier que les médecins militaires de la Suède avaient manifesté le désir de former une société, dans le but de pouvoir étudier et résondre ensemble les questions relatives au service de santé militaire, et que, de plus, cetle société, une fois formée, comptait publier une revue que l'Association pourrait utiliser, le Comité central qui, jusqu'ici n'a pas eu d'organe pour la publication d'articles sur le service de santé, décida, en se fondant sur des essais faits avec succès dans d'autres pays, qu'il fallait saisir l'occasion qui se présentait de combler cette lacune. Le Comité résolut en outre de contribuer, par une allocation annuelle de 400 krones (fr. $554^{1}$ ) à la revue projetée. La Société des médecins militaires de Suède s'est constituée dès lors, et la revue sera publiée ${ }^{2}$.

Le Comité des dames, qui est affilié à l'Association, a préparé et confectionné une partie du linge que la générosité de Sa Majesté le Roi a, l'an dernier, offert à l'Association. Sa Majesté la Reine douairière a daigné, cette année, offrir à l'Association divers effets de pansement en toile.

Le capital de l'Association qui, l'an dernier, à pareille époque, s'élevait à 23,000 krones (fr. 31,855), monte aujourd'hui à 26,236 lirones, 32 ôres (fr. 36,268). Les cotisations annuelles des membres

\section{Krones $=$ fr. $1385 \mathrm{c}$.}

2 Nous avons effectivement reçu la première livraison de ce recueil, qui vient accroitre le nombre des publications périodiques se rattachant directement à notre cuvre. On en trouvera le sommaire p. 72 .

Il est intéressant de remarquer, à cette occasion, le rapprochement qui tend à s'opérer entre les médecins militaires et les sociétés de la Croix rouge. On sait qu'à l'origine c'était en grande partie des rangs du personnel du service de santé officiel que partaient les objections au fonctionnement des sociétés de secours; aussi n'enregistrons-nous pas sans une légitime satisfaction un fait prouvant que cet antagonisme a complétement cessé en Suède. Ailleurs, la même tendance se manifeste très-généralement, mais nulle part on n'avait encore vu le corps médical et la Société de la Croix rouge s'unir pour la publication en commun de leurs travaux. C'est un véritable progrès et un exemple que nous ne saurions trop recommander à l'imitation des autres comités centraux.

(Note du Comite international.) 
et les dons particuliers se sont élevés à 3,312 krones (fr. 4,557, 22). Le Comité a encore à faire connaître que la bibliothèque de l'Association s'est enrichie, cette année comme les précédentes, de divers dons, témoignant tous de l'intérêt que l'on porte à notre cuvre.

Les dernières guerres ont suffisamment prouvé que les associations de secours - et il s'en est constitué maintenant dans presque tous les pays - ne sauraient dèsormais faire défaut sans grand inconvénient, et que d'ailleurs, en temps de guerre, le service de santé officiel, même dans les pays les plus prospères où il est le mieux organisé, ne peut, sans le secours de nos associations, répondre d'une manière satisfaisante à toutes les exigences. Le Comité croit donc devoir exhorter, de la manière la plus pressante, tout palriote à ne jamais se lasser d'accorder son intérêt et sa bienveillance à notre Association, afin que si, à l'heure du danger, nos services sont demandés pour le pays, l'Association soit à même de fournir des soins et des consolations aux compatriotes qui versent leur sang pour leur patrie.

Stockholm, le $\mathcal{L}^{\text {er }}$ novembre 1875.

$$
\text { Le Président, }
$$

A.-G. Laejonhufved.
Le Secrétaire, Axel von Heyne. " 